\title{
Vitrification of immature oocytes of goats in Bangladesh
}

\author{
MN Sharif, SM Choudhury, MM Rahman, MM Rahman'1, NS Juyena and \\ MMU Bhuiyan* \\ Department of Surgery and Obstetrics, Faculty of Veterinary Science, Bangladesh \\ Agricultural University, Mymensingh-2202, Bangladesh
}

\begin{abstract}
Cryopreservation of oocytes and embryos by vitrification can have advantages in assisted reproductive technologies (ARTs) in mammals. The aim of this study was to establish an effective vitrification procedure and cryodevice for goat's oocytes in Bangladesh. Cumulus oocyte complexes (COCs) were collected from ovaries from slaughterhouse. COCs with more than 3 layers of cumulus cells were selected. COCs were vitrified by two-step procedure using $7.5 \%$ and $15 \%$ dimethyl sulphoxide (DMSO) as cryoprotective agent (CPA), loaded on Cryotop or French mini-straw, then directly plunged into liquid nitrogen (LN2). Then the COCs containing Cryotop or French mini-straws were warmed in $0.25 \mathrm{M}$ sucrose and 20\% FBS-supplemented tissue culture medium (TCM) 199 followed by in vitro culture in $50 \mu \mathrm{l}$ droplets of bicarbonate-buffered TCM 199 supplemented with $10 \%$ FBS, pyruvate, FSH and oestradiol for $24 \mathrm{~h}$ at $39^{\circ} \mathrm{C}$ with $5 \% \mathrm{CO}_{2}$ in humidified air. After maturation culture, oocytes were denuded and examined under inverted microscope for presence of polar body as the indication of maturation. The in vitro maturation rate of goat's oocytes after vitrification and warming was $39.3 \pm 6.8 \%, 31.3 \pm$ $9.4 \%, 61.6 \pm 14.2 \%$ when using Cryotop (cryodevice), French mini-straws and without vitrification (control), respectively. Maturation rate was significantly higher $(\mathrm{P}<0.05)$ without vitrification. It is suggested that both Cryotop and French mini-straw are efficient cryodevices for vitrification of goat's oocytes and further investigation is required to optimize the protocol for vitrification and warming procedure for the satisfactory survival of goat's oocytes. (Bangl. vet. 2018. Vol. 35, No. 1\&2, 7 - 12)
\end{abstract}

\section{Introduction}

The fertility of an animal can be increased by assisted reproductive technologies (ARTs) such as in vitro maturation (IVM), fertilization (IVF) and culture (IVC) of embryos followed by transfer. For production of embryos using ARTs, cryopreservation of oocytes and embryos have great advantages.

Vitrification is a process of cryopreservation during which solidification of a solution occurs without the formation of ice crystals (Chian et al., 2004). Successful vitrification of mammalian oocytes may increase the success of ARTs. Vitrification has a practical importance in IVM, IVF, IVC, nuclear transfer and embryo preservation. After the technique was first used in embryology by Rall and Fahy (1985) on mouse embryos,

\footnotetext{
${ }^{1}$ Upazila Livestock Officer, Department of Livestock Services (DLS), Bangladesh

*Corresponding author:- E-mail: mmubhuiyan@gmail.com
}

DOI: https://doi.org/10.3329/bvet.v35i1-2.53382 
considerable advances have been made and offspring have been produced using frozen-thawed oocytes in cows (Vieira et al., 2002), horses (Maclellan et al., 2002), goats (Purohit et al., 2012) and humans (Chian et al., 2009). However, no attempt on vitrification of oocytes or embryos has been made in Bangladesh.

A wide variety of approaches are used to improve methods of oocyte vitrification. The majority of studies examined the effects of various cryoprotective compounds, and compared different cooling and warming conditions (McGrath, 1997; Horvath et al., 2008). However, cryodevice used for vitrification is important for successful outcome of cryopreservation. In oocyte vitrification, open pulled straws (OPS; Vajta and Nagy, 2006), solid surface vitrification (SSV; Dinnyes et al., 2000) and Cryotops (Chian et al., 2004) have been reported as successful cryodevices. However, no precise report on cryoprotectant and cryodevice is available in Bangladesh. Therefore, the present study was conducted to establish an effective vitrification procedure of goat's oocytes in Bangladesh and to determine an effective cryodevice.

\section{Materials and Methods}

\section{Chemicals and reagents}

Chemicals, reagents and media constituents were purchased from Sigma-Aldrich Chemicals, St Louis, USA and Sigma-Aldrich Chemic Gmbh, Steinheim, Germany. Media were prepared under standard protocol following aseptic technique. The media used for IVM and vitrification of oocytes were filtered using a disposable filter (0.22 $\mu \mathrm{m}$ pore size membrane filter, Durapure membrane filter, Ireland). The IVM media were routinely equilibrated at $37^{\circ} \mathrm{C}$ with $5 \% \mathrm{CO}_{2}$ in humidified air for at least 1 $\mathrm{h}$ before use.

\section{Oocyte recovery and culture}

Ovaries of goats were collected from the local slaughterhouse and transported to the laboratory in a vacuum flask containing warm saline $(0.9 \%, \mathrm{w} / \mathrm{v}$, sodium chloride, at $35-37^{\circ} \mathrm{C}$ ) within 2 to $3 \mathrm{~h}$ of slaughtering. In the laboratory, ovaries were rinsed 3 times in saline at $37^{\circ} \mathrm{C}$ before aspiration of follicles. Follicles of $2-8 \mathrm{~mm}$ diameter were aspirated using an 18-gauge needle attached to a $10-\mathrm{mL}$ disposable plastic syringe (Sterio pack Disposable Syringe, Opso Saline Ltd., Dhaka, Bangladesh). The follicular fluid was transferred in a $60 \mathrm{~mm}$ petri dish (Bio Basic Inc., Markham, Canada) and left for $5 \mathrm{~min}$ for sedimentation. The retrieved follicular aspirate was diluted with HEPESbuffered TCM 199 supplemented with bovine serum albumin (BSA) (washing medium). Oocytes were selected under a stereomicroscope (Labomed Inc, USA). The cumulus-oocyte-complexes (COCs) with more than three compact cumulus cell layers and homogenous ooplasm were selected for maturation. The COCs were washed three times in washing medium. 


\section{Vitrification of oocytes}

The COCs were washed twice in holding medium (HM) (Hepes-buffered TCM supplemented with $20 \%$ fetal bovine serum (FBS; Gibco ${ }^{\circledR}$, Invitrogen, NY, USA) and kept there for about $15 \mathrm{~min}$. The COCs were kept in the vitrification medium 1 (HM supplemented with 7.5\% DMSO) for 35-40 sec and then transferred to the vitrification medium 2 (HM supplemented with 15\% DMSO and $0.5 \mathrm{M}$ sucrose) for a further $25 \mathrm{sec}$. Vitrification was done using two-step procedure using either $0.25 \mathrm{ml}$ French ministraw or Cryotop (Kitazato Supply Co, Fujinomiya, Japan) as cryodevice. By using mouth-controlled pipette, 2 oocytes were loaded on the film strip of a Cryotop from vitrification medium 2 under a stereomicroscope. Before vitrification, almost all solution was removed to leave only a thin layer covering the oocytes on Cryotop. The Cryotop was immediately submerged into liquid nitrogen vertically. During loading COCs into the straws, warming solution - 1 (WS - 1; HM supplemented with $0.25 \mathrm{M}$ sucrose) was taken into the straw followed by filling with air. Then, 3-5 COCs was loaded into the straw with least volume of vitrification medium 2 . Then air was taken again followed by filling with warming solution -1. Finally, small amount of air was taken into the straw and submerged into the $\mathrm{LN}_{2}$ for vitrification.

\section{Warming and in vitro maturation (IVM) of oocytes}

After removal of the COCs containing cryodevice (Cryotop/French mini-straw) from $\mathrm{LN}_{2}$, COCs were inserted directly into warming solution -1 for 5 to $10 \mathrm{~min}$. Then the oocytes were transferred into warming solution - 2 (WS - 2; HM supplemented with $0.125 \mathrm{M}$ sucrose) for $5 \mathrm{~min}$. Finally, the COCs were washed twice in HM followed by washing once in maturation medium before culture in vitro. The basic medium for oocyte maturation was bicarbonate-buffered TCM 199 supplemented with $0.25 \mathrm{mM}$ pyruvate, $10 \%$ FBS, $0.05 \mu \mathrm{g} / \mathrm{ml} \mathrm{FSH}$ and $1.00 \mu \mathrm{g} / \mathrm{mL}$ oestradiol. Fifty $\mu \mathrm{L}$ droplets of maturation medium were prepared in a $35 \mathrm{~mm}$ petri dish (Greiner Bio_One, Frickenhausen, Germany) and covered with mineral oil. For in vitro maturation, 5 to 10 COCs were cultured in each drop of medium for $24 \mathrm{hrs}$ at $39^{\circ} \mathrm{C}$ with $5 \% \mathrm{CO}_{2}$ in humidified air.

\section{Evaluation of maturation of oocytes}

After $24 \mathrm{~h}$ of maturation culture, oocytes were denuded using 3\% (w/v) sodium citrate in Hepes-buffered TCM 199 under stereomicroscope. The cumulus-free oocytes were examined for polar body extrusion under inverted microscope (Leica DM IRB) with the help of mouth-controlled pipette. The oocytes were regarded as matured when extrusion of $1^{\text {st }}$ polar body was observed under zona pellucida.

\section{Experimental approaches and statistical analysis}

To determine an effective cryodevice for vitrification of immature oocytes of goats, COCs were vitrified using either Cryotop or French mini straw. The immature COCs without vitrification were used as control. The data on rate of oocyte maturation were expressed as mean \pm SD. The data were analysed by using non-parametric one-way 
ANOVA followed by Bartlett's test for comparison using Graphpad PRISM software version 5.0. The variation was considered significant when the $P$ value was less than 0.05 .

\section{Results and Discussion}

This study was done to establish an effective vitrification procedure of goat's oocytes and to determine an effective cryodevice. The efficacy of vitrification was determined by evaluation of post-vitrification maturation on the basis of presence of extrusion of first polar body in the oocytes.

Table 1 shows that the IVM rate of oocytes of goats after vitrification and warming was $39.3 \pm 6.8$ when Cryotop was used as cryodevice and $31.3 \pm 9.4 \%$ with French mini-straw. The difference was not significant. The IVM rate was $61.6 \pm 14.2 \%$ when culture was done without vitrification (control). The difference between vitrified and control groups was significant $(\mathrm{P}<0.5)$.

Table 1: Comparis on of maturation rate of immature oocytes between two cryodevices after vitrification and warming

\begin{tabular}{l|c|c|c}
\hline \multicolumn{1}{c|}{ Cryodevice } & $\begin{array}{c}\text { No. of oocytes } \\
\text { vitrified }\end{array}$ & $\begin{array}{c}\text { No. of oocytes } \\
\text { matured }\end{array}$ & $\begin{array}{c}\text { Maturation rate } \\
(\%)\end{array}$ \\
\hline Cryotop & $40(\mathrm{n}=5)$ & 15 & $39.3 \pm 6.8^{\mathrm{b}}$ \\
Straw & $51(\mathrm{n}=4)$ & 16 & $31.3 \pm 9.4^{\mathrm{b}}$ \\
No vitrification (control) & $92(\mathrm{n}=9)$ & 57 & $61.6 \pm 14.2^{\mathrm{a}}$ \\
\hline
\end{tabular}

Percentage values are mean \pm SD. $n=$ number of replicates.

$\mathrm{a}, \mathrm{b}$ Values with superscripts within same column differed significantly from each other $\mathrm{P}<0.05)$.

The IVM rate of oocytes of goats after vitrification and warming was lower than without vitrification (control) (31.3 to $39.3 \%$ vs. $61.6 \%$, respectively). Similarly, lower rate of IVM of goat oocytes after vitrification and warming was reported in India than non-vitrified control (Purohit et al., 2012). Moreover, lower rates of IVM of bovine oocytes after vitrification and warming were reported in Turkey by Cetin and Bastan (2006) and in Malaysia by Hajarian et al. (2011). Two major obstacles that may reduce success of vitrification are ice crystal formation and chilling injury to the oocytes (Vajta and Kuwayama, 2006). Chilling injury mostly affects the cytoskeleton and cell membranes of oocytes, resulting in lower maturation rate than non-vitrified control (Morato et al., 2008). This indicates that the protocol for vitrification of oocytes of goats and cows needs to be improved.

In vitro maturation rate of goat's oocytes without vitrification is similar to that reported in Bangladesh (Khatun et al., 2011) and India (Purohit et al., 2012). Contrasting to the present finding, higher rates of in vitro maturation (71.4 to 81.4\%) were reported in goat oocytes when cultured without vitrification elsewhere (Izquierdo et al., 2002; Anguita et al., 2007). The variation in maturation rates in 
oocytes of goats may be due to variations in culture period. Goat's oocytes were cultured for $24 \mathrm{~h}$ compared to $27-28 \mathrm{~h}$ by other investigators. Higher rates of IVM of goat oocytes have been reported when oocytes were cultured for $27 \mathrm{~h}$ than for $24 \mathrm{~h}$ (Rho et al., 2001). However, Agrawal et al. (1995) and Samake et al. (1999) suggested that goat's oocytes can be successfully matured in vitro by culturing for 24 to $27 \mathrm{~h}$.

Contrasting to the present finding, Cryotop has been reported superior to other cryodevices when IVM of bovine oocytes was done using open pulled straw (OPS), Cryotop and electro microscopic grid (Hajarian et al., 2011). No precise reports in goats are available to compare the efficacy of cryodevices for vitrification of immature oocytes in Bangladesh. Moreover no vitrified oocytes in buffaloes matured when Cryotop was used as cryodevice in Bangladesh (Akter, 2015). This means, the present investigation for vitrification of oocytes of goats in Bangladesh was encouraging. Further studies are required to optimize the protocol for vitrification of immature goats' oocytes. In conclusion, both Cryotop and French mini-straw are efficient as cryodevices for vitrification of goat oocytes. Further investigation is required to optimize the protocol for vitrification and warming of goat oocytes.

\section{Acknowledgement}

The research work was funded by Higher Education Quality Enhancement Project (HEQEP) of University Grants Commission of Bangladesh (CP 3015).

\section{References}

Agrawal KP, Shama T, Sexena C, Sharma N 1995: Chronology of first meiotic events of caprine oocytes matured in vitro. Indian Journal of Animal Sciences 65 285-288.

Akter S 2015: Effect of sucrose and equilibration time on in vitro maturation of vitrified buffalo oocytes. MS in Animal Science Thesis, Department of Animal Science, Faculty of Animal Husbandry, Bangladesh Agricultural University, Mymensingh, Bangladesh pp. 68.

Anguita B, Jimenez-Macedo AR, Izquierdo D, Mogas T, Paramio MT 2007: Effect of oocyte diameter on meiotic competence, embryo development, p34 (cdc2) expression and MPF activity in prepubertal goat oocytes. Theriogenology 67 526-536.

Cetin Y, Bastan A 2006: Cryopreservation of immature bovine oocytes by vitrification in straws. Animal Reproduction Science 92 29-36.

Chian RC, Huang JYJ, Gilbert L, Son WY, Holzer H, Cui SJ, Buckett WM, Tulandi T, Tan SL 2009: Obstetric outcomes following vitrification of in vitro and in vivo matured oocytes. Fertility and Sterility 91 2391-2398.

Chian RC, Kuwayama M, Tan L, Tan J, Kato O, Nagai T 2004: High survival rate of bovine oocytes matured in vitro following vitrification. Journal of Reproduction and Development 50 685-696. 
Dinnyes A, Dai Y, Jiang S, Yang X 2000: High developmental rates of vitrified bovine oocytes following parthenogenetic activation, in vitro fertilization and somatic cell nuclear transfer. Biology of Reproduction 63 513-518.

Hajarian H, Wahid H, Rosnina Y, Daliri M, Dashtizad M, Mirzapour T, Yimer N, Bukar MM, Iswadi MI, Mazni OA 2011: Cryotop device enhances vitrification outcome of immature bovine oocytes. Journal of Animal and Veterinary Advances 10 2541-2545.

Horvath G, Seidel Jr GE 2008: Use of fetuin before and during vitrification of bovine oocytes. Reproduction in Domestic Animals 43 333-338.

Izquierdo D, Villamediana P, Lopez-Bejar M, Paramio MT 2002: Effect of in vitro and in vivo culture on embryo development from prepubertal goat IVM-IVF oocytes. Theriogenology 57 1431-1441.

Khatun M, Bhuiyan MMU, Ahmed JU, Haque A, Rahman MB, Shamsuddin M 2011: In vitro maturation and fertilization of prepubertal and pubertal black Bengal goat oocytes. Journal of Veterinary Science 12 75-82.

Maclellan LJ, Carnevale EM, Coutinho da Silva MA, Scoggin CF, Bruemmer JE, Squires EL 2002: Pregnancies from vitrified equine oocytes collected from super-stimulated and non-stimulated mares. Theriogenology 58 911-919.

McGrath JJ 1997: Quantitative measurement of cell membrane transport: technology and applications. Cryobiology 34 315-334.

Morato R, Izquierdo D, Paramio MT, Mogas T 2008: Cryotops versus open-pulled straws (OPS) as carriers for the cryopreservation of bovine oocytes: effects on spindle and chromosome configuration and embryo development. Cryobiology 57 137-141.

Purohit GN, Meena H, Solanki K 2012: Effects of vitrification on immature and in vitro matured, denuded and cumulus compact goat oocytes and their subsequent fertilization. Journal of Reproduction and Infertility 13 53-59.

Rall WF, Fahy GM 1985: Ice-free cryopreservation of mouse embryos at -196 degrees $C$ by vitrification. Nature 313 573-575.

Rho GJ, Hahnel AC, Betteridge KJ 2001: Comparisons of oocyte maturation times and three methods of sperm preparation for their effects on the production of goat embryos in vitro. Theriogenology 56 503-516.

Samake S, Amoah EA, Mobini S, Gazal O, Gelaye S 1999: In vitro fertilization of goat oocytes during the non-breeding season. Small Ruminant Research 35 49-54.

Vajta G, Nagy ZP 2006: Are programmable freezers still needed in the embryo laboratory? Review on vitrification. Reproductive Biomedicine Online 12 779-796.

Vieira AD, Mezzalira A, Barbieri DP, Lehmkuhl RC, Rubin MIB, Vajta G 2002: Calves born after open pulled straw vitrification of immature bovine oocytes. Cryobiology 45 91-94. 